\title{
EchoGéo
}

40 | 2017

Ressources urbaines (2)

\section{Accéder au foncier et à l'immobilier à Mexico. La diversité des marchés vue depuis l'espace public}

\section{Antonine Ribardière and Jean-François Valette}

\section{(2) OpenEdition \\ 1 Journals}

Electronic version

URL: https://journals.openedition.org/echogeo/14951

DOI: 10.4000/echogeo.14951

ISSN: 1963-1197

\section{Publisher}

Pôle de recherche pour l'organisation et la diffusion de l'information géographique (CNRS UMR 8586)

\section{Electronic reference}

Antonine Ribardière and Jean-François Valette, "Accéder au foncier et à l'immobilier à Mexico. La diversité des marchés vue depuis l'espace public", EchoGéo [Online], 40 | 2017, Online since 21 June 2017, connection on 10 August 2021. URL: http://journals.openedition.org/echogeo/14951 ; DOI: https://doi.org/10.4000/echogeo.14951

\section{This text was automatically generated on 10 August 2021}

EchoGéo est mis à disposition selon les termes de la licence Creative Commons Attribution - Pas d'Utilisation Commerciale - Pas de Modification 4.0 International (CC BY-NC-ND) 


\title{
Accéder au foncier et à l'immobilier à Mexico. La diversité des marchés vue depuis l'espace public
}

\author{
Antonine Ribardière and Jean-François Valette
}

1 L'accès au sol et au toit urbains constitue une dimension majeure de la question des ressources urbaines, quels que soient l'échelle et les espaces considérés dans la ville. Le marché foncier et immobilier peut être analysé sous l'angle de la géographie des valeurs et des formes de la division sociale de l'espace urbain qu'il révèle (Mouillart, 1998 ; Renard, 2003 ; Boulay, Guérois et Le Goix, 2011 ; Ribardière et Valette, 2017) ; sous l'angle de la construction de la valeur «d'opinion » et du poids des représentations dans les qualifications des contextes résidentiels (Halbwachs, 1908); sous celui du rapport au cadre légal et formel de la production résidentielle et de l'occupation du logement (Soares Gonçalves, 2017) ; mais aussi, en amont, sous l'angle de l'accès que les citadins peuvent avoir au marché.

2 Une des conditions de l'accès au marché foncier et immobilier passe par l'information elle-même sur ce marché : à Mexico, comme dans la plupart des agglomérations, au Nord comme au Sud, les canaux d'information prennent des formes plurielles, qu'il s'agisse des réseaux sociaux de connaissances ou du «bouche-à-oreille », des annonces papier, ou encore de la publication d'annonces en ligne.

3 Ces différents canaux sont particulièrement visibles depuis l'espace public : la rue ellemême constitue un espace d'information, qui relaie (au moins partiellement) les différentes filières d'accès au logement - qu'il s'agisse de terrains ou de biens immobiliers, de ventes ou de locations. Les affiches "publicitaires ", artisanales ou à l'enseigne d'un grand groupe, directement apposées sur les façades ou sur du mobilier urbain, dans les espaces situés le long d'axes de forte circulation, constituent une source de renseignements précieux, exploitables à une échelle locale ${ }^{1}$. Ces affiches se situent «à mi-chemin » entre les réseaux familiaux et amicaux, pour lesquels le lien 
social est discriminant, et les sources classiques d'annonces dans les journaux papier ou sur Internet, davantage exploitables à l'échelle de l'ensemble de l'agglomération. En tant que relais des différents segments du marché, formels et informels, les affiches constituent une entrée pour comprendre le fonctionnement et les enjeux associés aux différentes filières d'accès aux marchés fonciers et immobiliers dans les divers contextes de peuplement de la zone métropolitaine de Mexico (ZMVM) ${ }^{2}$.

4 Les photographies présentées ont été prises lors de séjours de terrain réalisés entre 2009 et 2015 dans la métropole mexicaine ${ }^{3}$ (voir annexe). Elles sont présentées par grand groupe de peuplement et par type de marché : d'abord, les colonies populaires nées dans l'informalité, correspondant aux deux tiers de l'espace construit, sont caractérisées par une forte visibilité du marché foncier, mais aussi par l'importance du marché locatif. Ensuite, les ensembles de logements sociaux légaux, autre forme majeure du logement des catégories populaires dans la métropole, sont le lieu d'un marché de la revente très actif. Enfin, les espaces des catégories moyennes et aisées renvoient a priori à l'hétérogénéité des canaux d'information sur le marché.

\section{Annonces affichées dans les colonies populaires : la part belle au marché foncier}

5 Comme en témoigne l'affichage d'annonces dans l'espace public, les colonies populaires, construites dans un cadre illégal - mais dont le statut a pu évoluer depuis les premières installations - constituent des espaces privilégiés de l'observation du dynamisme du marché foncier et immobilier au sein de la ZMVM. Dans le cas de Milpa Alta, La Paz, ou encore Tláhuac (illustrations 1 à 6), l'illégalité est encore prégnante, qu'il s'agisse de l'usage du sol - résidentiel dans les faits, mais agricole selon les plans d'urbanisme -, ou de la propriété foncière - dans un régime mexicain laissant encore une large part à un statut de propriété collective spécifique ${ }^{4}$. Ainsi, la question des prix du foncier et de l'immobilier relève d'une problématique très délicate dans ces zones où l'urbanisation est prohibée, mais où la pression est importante.

6 Les annonces affichées dans l'espace public, imprimées ou réalisées manuellement, comportent les caractéristiques principales du bien ou du terrain à vendre ou à louer : superficie, nom de la colonie (quartier), prix et numéro de téléphone à contacter, etc. Ces affiches témoignent des différences importantes entre les quartiers étudiés en termes de valeurs foncières et immobilières, d'équipement du lot (connexion aux services de base, sécurité foncière) et de dynamisme d'ensemble du marché local.

7 À San Pablo Oztotepec, village en cours d'expansion situé dans la délégation de Milpa Alta au sud de la Ciudad de México (CDMX), le marché immobilier semble assez peu dynamique par rapport aux zones plus proches de la ville : le marché est globalement endogène, dans le sens où les réseaux de connaissances locaux sont privilégiés, dans un contexte marqué par «l'héritage villageois » et l'enclavement par rapport aux axes de transport vers le reste de la ville (trois heures de transport en commun sont nécessaires pour rejoindre le centre). En revanche, le marché foncier est quant à lui plus visible(illustration 1): les affiches annonçant des ventes de terrains sont nombreuses, malgré l'illégalité des transactions et le poids des réseaux de la comunidad dans l'instauration d'une relative omerta au sujet de ces marchés. En 2014, les valeurs du mètre carré de terrain sont parmi les plus basses de la CDMX ${ }^{5}$. 
Illustration 1 - Vente de terrains à San Pablo Oztotepec, Milpa Alta, CDMX

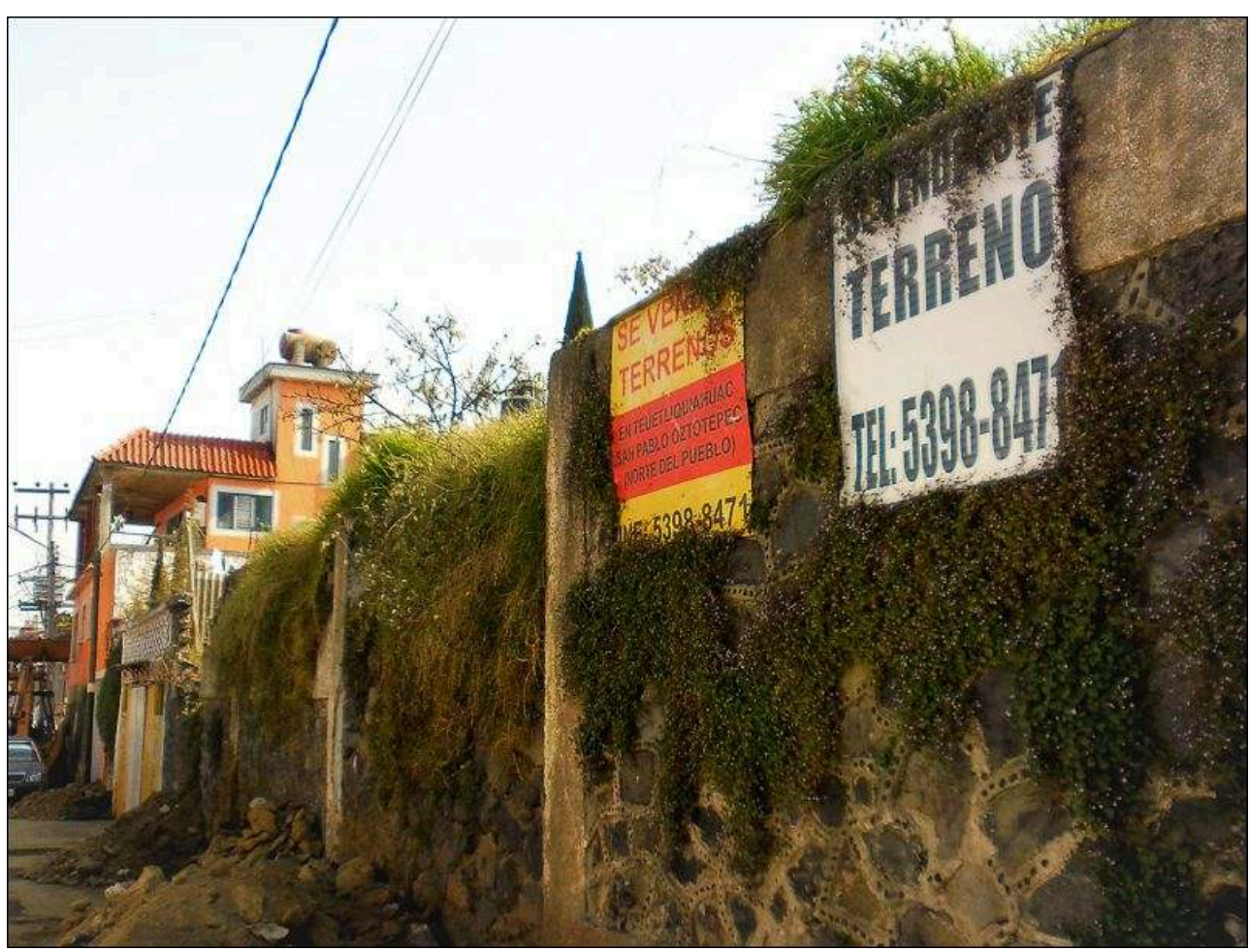

Auteur : J.-F. Valette, novembre 2014

8 La faible visibilité du marché immobilier par rapport au marché foncier est aussi remarquable dans le cas des colonies populaires de Tláhuac. Cette zone périphérique du sud-est de la ville en cours de densification a connu une augmentation des valeurs immobilières et foncières forte depuis la fin des années 2000 , en raison de la meilleure connexion au reste de la ville avec l'arrivée d'une nouvelle ligne de métro ${ }^{6}$. La vente de biens construits transparaît peu dans l'espace public, en revanche beaucoup de transactions sont en cours pour des terrains, notamment par l'intermédiaire de réunions d'associations locales de propriétaires. 


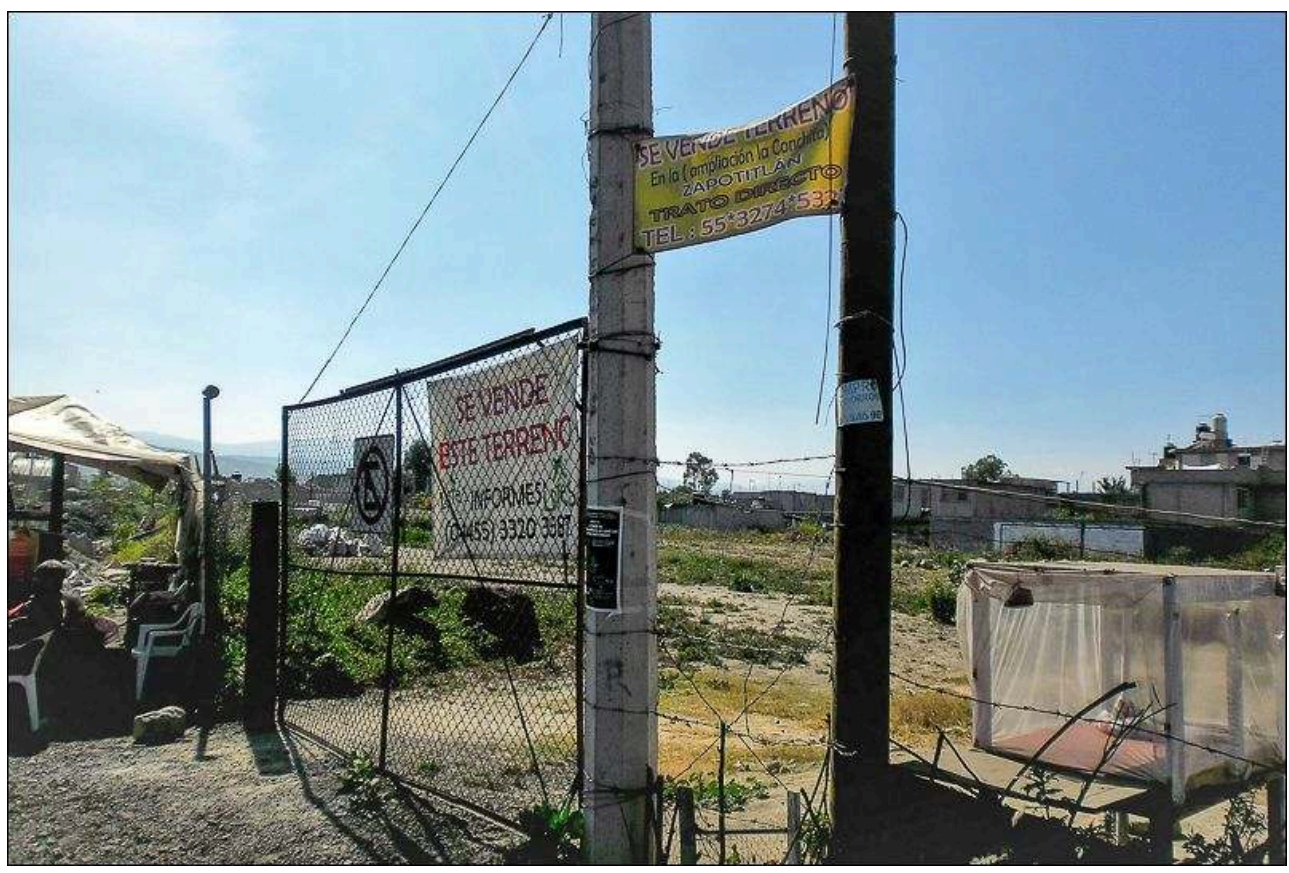

Auteur : J.-F. Valette, novembre 2014

Dans l'État de Mexico cette fois, la municipalité de La Paz s'est peuplée le long d'un front d'urbanisation sur les collines orientales de la métropole. Banlieues des anciennes banlieues de Nezahualcóyotl - le «plus grand bidonville du monde » des années 1980, aujourd'hui consolidé -, ces colonies connaissent actuellement une densification rapide. La pression foncière y est très forte depuis une vingtaine d'années. Dans ces contextes, seule une minorité de résidents sont en règle et les conflits fonciers sont nombreux au sein de la communauté ejidale (membres de l'ejido), entre les propriétaires qui vendent leurs terrains et participent à l'urbanisation de la zone, et ceux qui veulent les conserver. La mise en garde d'une partie des membres de l'ejido telle qu'elle apparait sur l'illustration 3 s'appuie sur les instruments juridiques existants, chargés de sanctionner les ventes frauduleuses (parquet général judiciaire - PGJ -, parquet spécialisé sur les questions foncières). Elle révèle également d'autres acteurs: en premier lieu l'IMEVIS, Institut de l'État de Mexico du Logement Social, organisme public chargé d'accompagner les familles dans les démarches de titrisation, et en second lieu, les différents niveaux de l'autorité étatique, comme la municipalité de La Paz et le gouvernement de l'État de Mexico, dont les intérêts électoraux pèsent dans les tolérances accordées aux nouveaux résidents - surtout si ces derniers sont organisés en groupes de pression. 
Illustration 3 - Affichage public d'un message de la part de la communauté locale

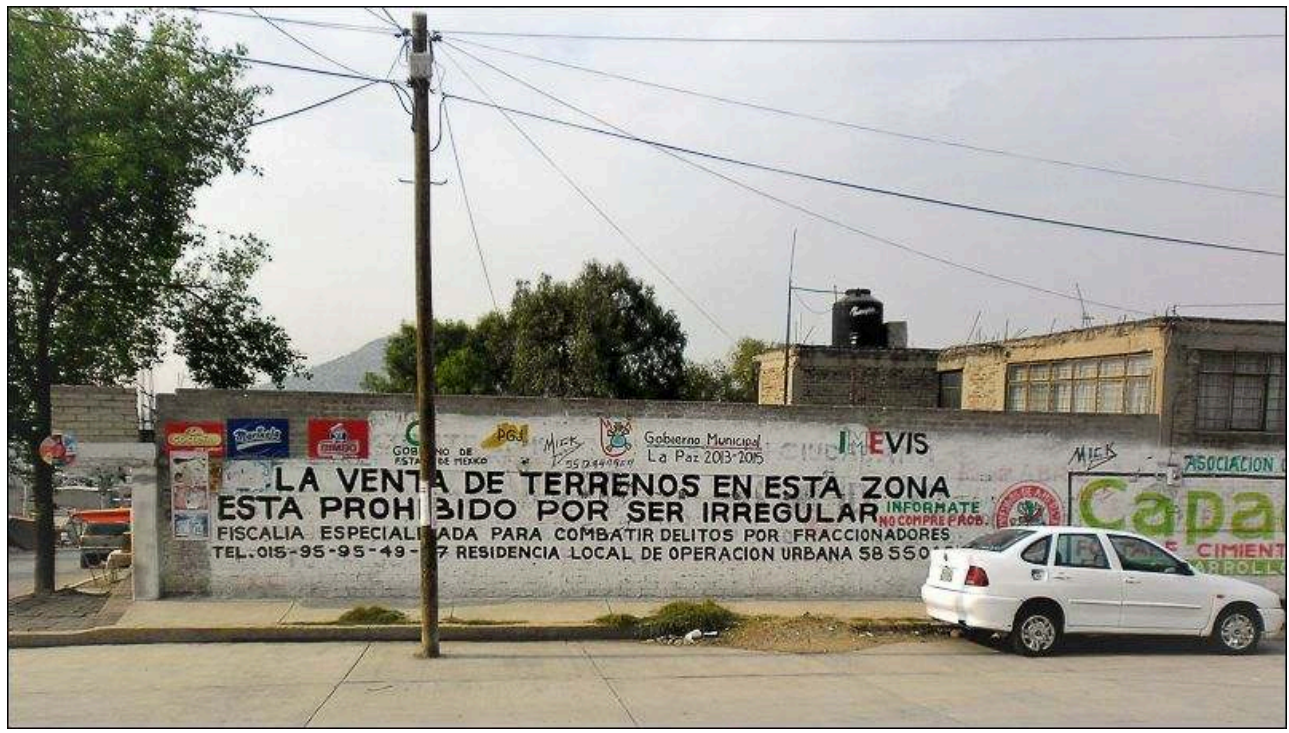

"La vente de terrains dans cette zone est interdite, car irrégulière. Informe-toi, n'achète pas des problèmes. Parquet spécialisé chargé de combattre les délits des lotisseurs ».

Auteur : J.-F. Valette, novembre 2014.

En négatif, un tel message exprime le dynamisme du marché foncier local. Quelques dizaines de mètres plus loin, les nombreuses affiches annonçant la vente de terrains tranchent avec cet apparent rappel à la loi (illustration 4). Ce dynamisme se nourrit de la demande, mais aussi de l'offre, plus importante dans une municipalité comme La Paz que dans les délégations de la CDMX. La disponibilité du sol renvoie aux superficies non construites, mais aussi au contexte juridique - l'État de Mexico offrant un cadre moins restrictif que l'entité capitale -, et enfin, dans le cas de La Paz, à l'absence de concurrence avec les groupes immobiliers constructeurs de lotissements géants de logements sociaux. 
Illustration 4 - Vente de terrains à La Paz

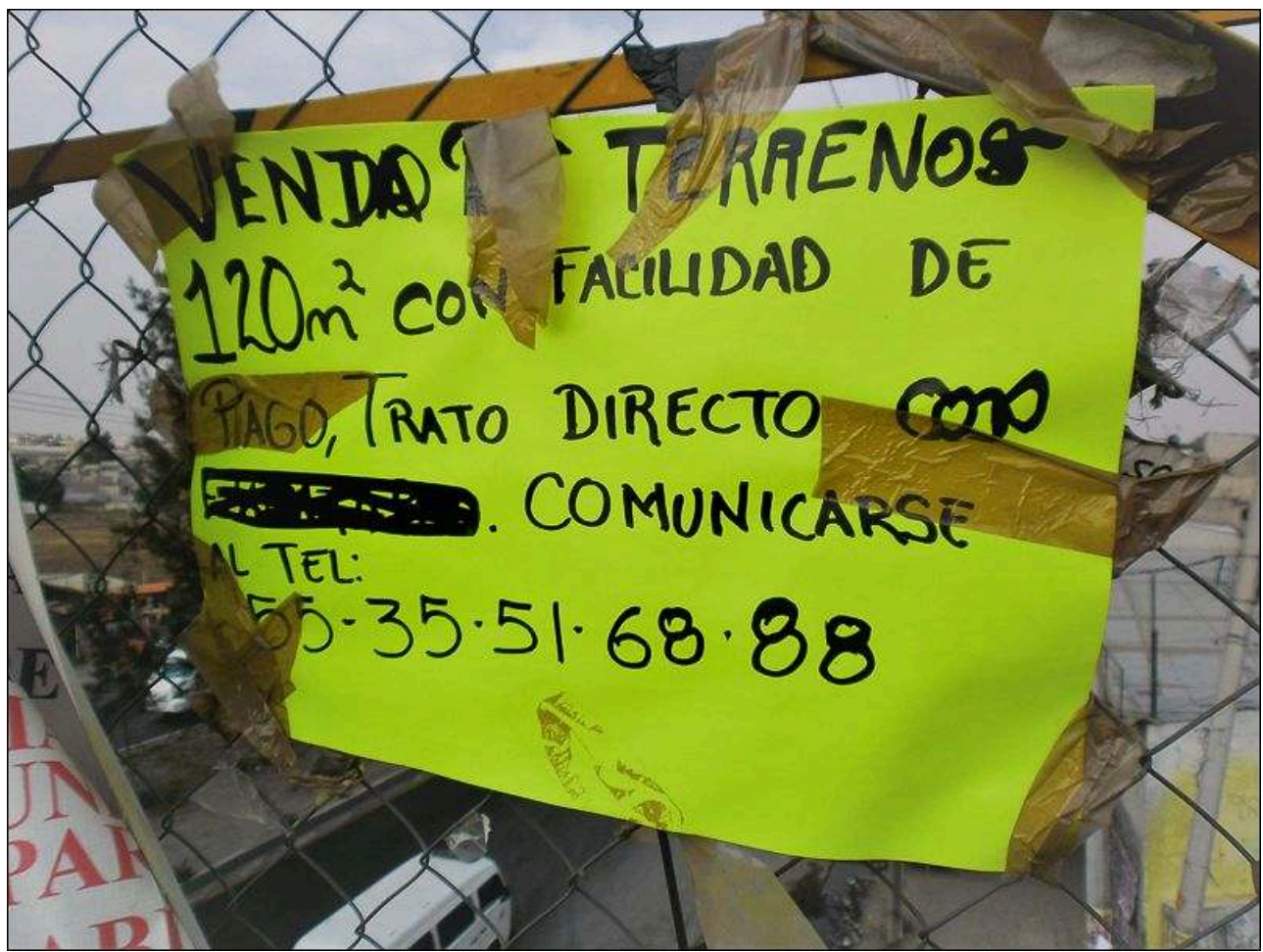

" Je vends $120 \mathrm{~m}^{2}$ avec facilités de paiement. Contact direct. Se faire connaître au (...) ».

Auteur : J.-F. Valette, novembre 2014

11 Pour autant, le marché immobilier n'est pas inexistant dans les colonies populaires. Toujours à La Paz, dans le quartier Jiménez Cantú urbanisé à partir de la fin des années 1970, l'illustration 5 témoigne de la mise en vente d'une maison, l'affiche étant directement apposée sur le bien. Si quelques maisons en vente sont vacantes, comme c'est le cas ici, d'autres en revanche sont toujours habitées, par des familles spéculant sur la valeur en constante augmentation avec l'arrivée progressive des services dans la zone. Pour une maison bien entretenue, tout équipée, d'une surface classique - celle d'une parcelle lotie $\left(120 \mathrm{~m}^{2}\right)$-, les valeurs peuvent largement dépasser $60000 €$ en 2014 . 
Illustration 5 - Vente d'une maison consolidée à Jorge Jiménez Cantú, La Paz, État de Mexico

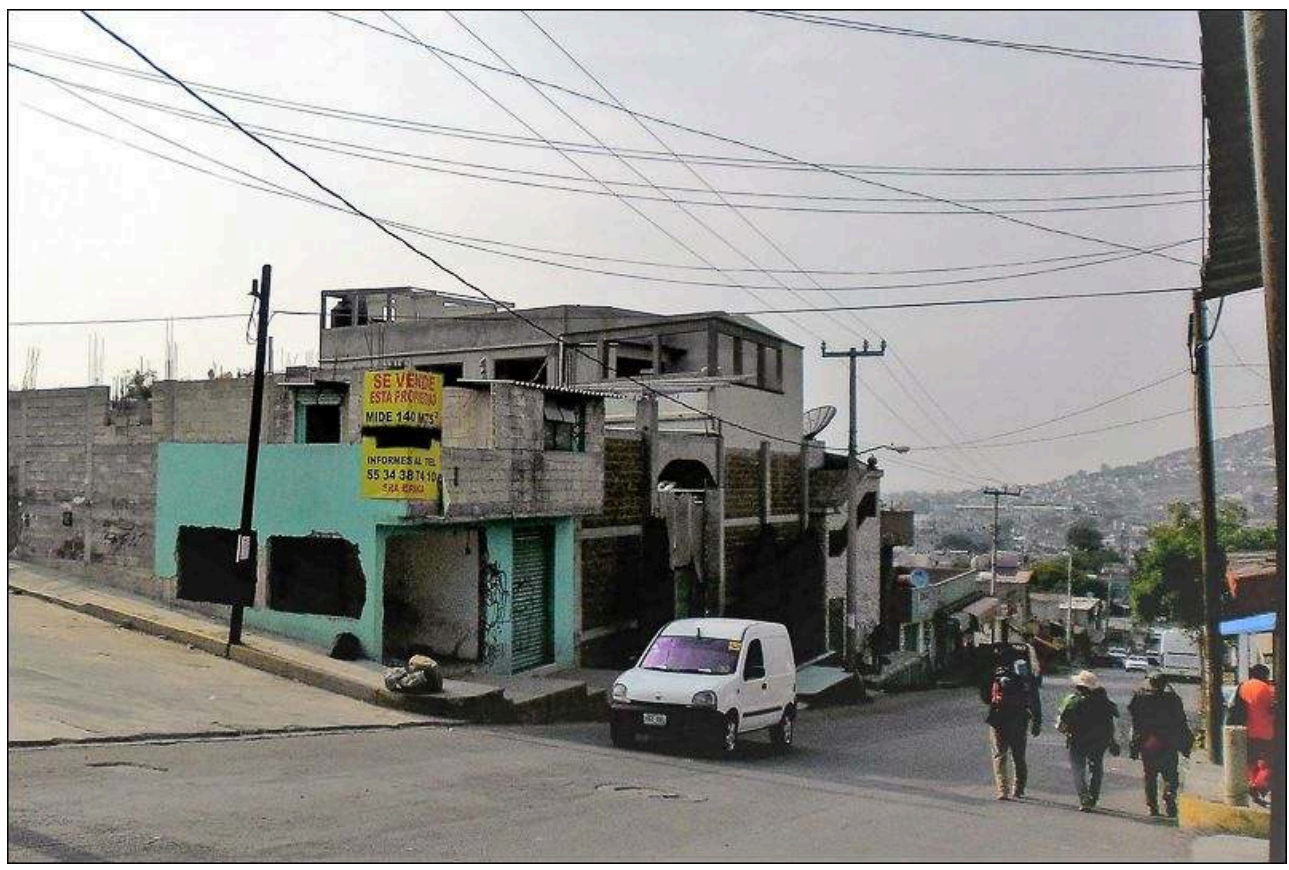

Auteur: J.-F. Valette, novembre 2014

\section{Le marché locatif très actif en colonie populaire}

Dans toutes les colonies populaires de notre échantillon, si le marché de la vente de biens reste discret dans l'espace public, le marché de la location est, quant à lui, remarquable. Il s'agit de chambres chez l'habitant, voire d'appartements, soit dans des maisons, soit dans des immeubles. Ainsi, la propriétaire de la maison photographiée sur l'illustration 6 met en location des chambres, dont la superficie varie entre 10 et $15 \mathrm{~m}^{2}$. La maison a été progressivement consolidée depuis l'arrivée en 1984 de la famille, qui a vécu dans les premières années dans des conditions extrêmement précaires. Désormais régularisée et équipée de la plupart des services urbains, la maison s'étend sur trois niveaux : en plus du logement des deuxième et troisième générations, l'aménagement de chambres a permis à la famille propriétaire d'augmenter ses revenus, par la location des chambres à des personnes extérieures. Comme l'explique la propriétaire : «ce sont des gens qui passent et qui demandent. Ils voient l'affiche devant la maison et demandent combien ça coûte, mais on ne les connaît pas ». Si les loyers sont souples, ils sont aussi moins élevés à La Paz que ceux pratiqués dans la CDMX, mais aussi dans d'autres périphéries de l'État de Mexico ${ }^{7}$. 
Illustration 6 - Affiche de location de chambres dans une maison consolidée à Jorge Jiménez Cantú, La Paz, État de Mexico

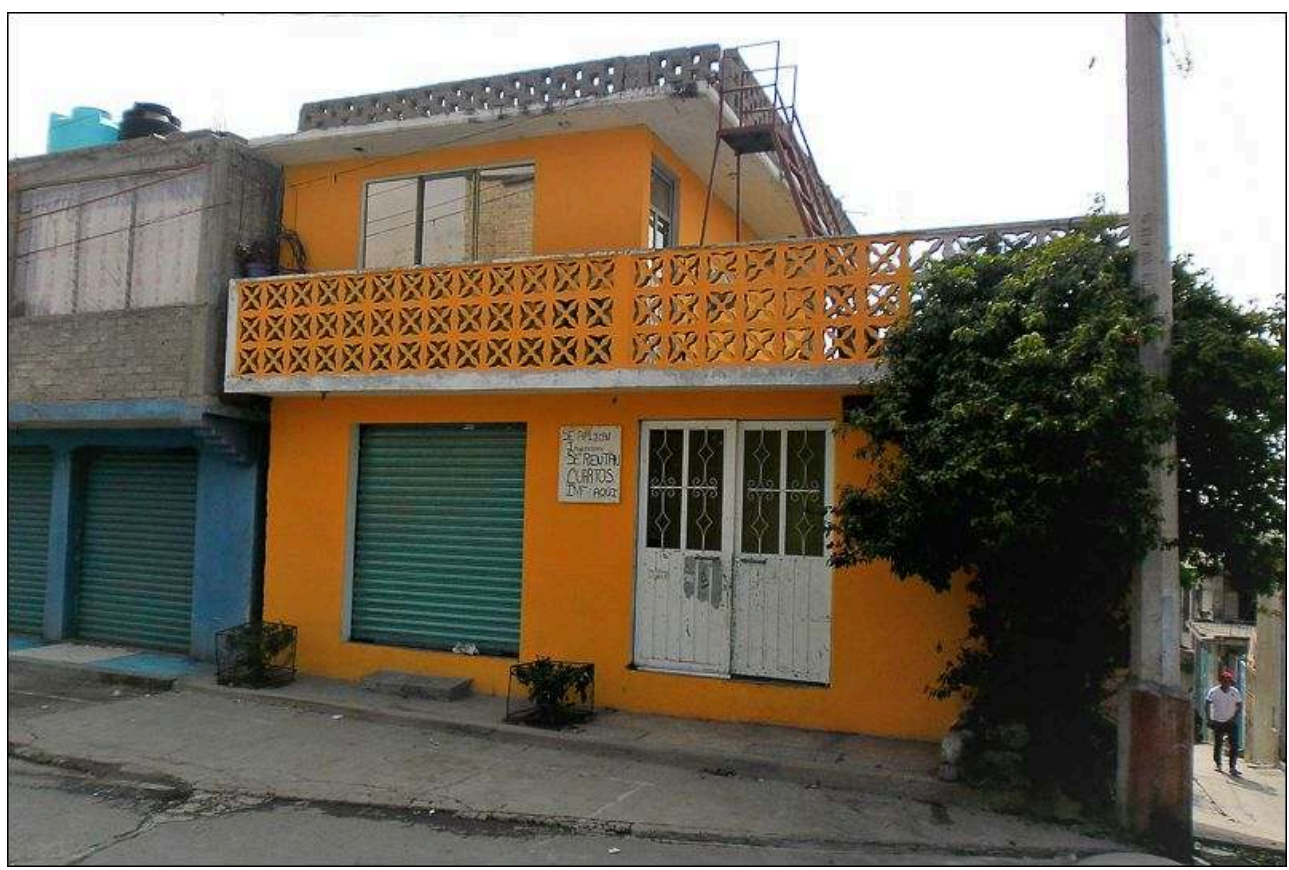

Auteur : J.-F. Valette, novembre 2014

L'apparente discrétion du marché immobilier à l'achat, par rapport au marché foncier ou au marché locatif, peut renvoyer à une série d'hypothèses. Premièrement, le marché immobilier est globalement considéré comme moins vif dans les colonies populaires par rapport aux autres portions de la ville (Kunz et al., Duhau, 2004), notamment dans les espaces les plus récemment urbanisés. L'enjeu premier se noue avant tout autour de l'accès au sol, puis dans des étapes ultérieures par la construction progressive, selon les moyens du ménage et de son entourage, de l'habitat. Deuxièmement, l'ensemble des investissements (physiques, moraux, financiers, à l'échelle des personnes et de leur entourage) mis en œuvre autour des dynamiques d'autoconstruction ${ }^{8}$ amène à lire les stratégies résidentielles d'accession à la propriété sur des temporalités potentiellement plus longues que dans d'autres parcs de logements (Hiernaux, 1991). Troisièmement, enfin, le marché immobilier existant donne la priorité aux réseaux de connaissances, plutôt qu'à l'affichage public. D'une manière générale, les entretiens ont révélé que dans les colonies populaires, le marché foncier et immobilier ne passe que très rarement par d'autres canaux que ceux liés aux réseaux de connaissances, amicales et/ ou familiales. Ces réseaux permettent le transfert direct des informations, l'instauration d'une relation de confiance dans un contexte de grande méfiance du fait de l'illégalité généralisée et de l'insécurité foncière, et une plus grande souplesse du crédit (négociations de l'apport et des mensualités). En revanche, le marché locatif apparait plus ouvert.

14 En dehors des colonies populaires, le marché du logement «du plus grand nombre » passe aussi par les ensembles résidentiels d'habitat social. 


\section{Circulation des biens dans les ensembles de logements sociaux : rente et recompositions de la population résidente}

Le modèle de constructions de logements sociaux horizontaux, sous la forme de petites maisons construites en série visant les catégories populaires capables d'accéder au crédit bancaire, est particulièrement présent dans les paysages de la périphérie de Mexico depuis une vingtaine d'années. À Cuautitlán, dans le nord de la métropole, la relative atonie du marché immobilier des colonies populaires tranche avec le dynamisme apparent de celui des ensembles de logements sociaux voisins (illustration 7) : parfois, comme dans le cas de cette rue fermée - fonctionnant sur le principe de la copropriété - ce sont même des réseaux internationaux d'agences immobilières qui s'emparent de ce marché, ici Century 21.

Illustration 7 - Affiche des services d'une agence Century 21 dans une rue fermée d'un lotissement en accession à la propriété aidée, Hacienda Cuautitlán, Cuautitlán, État de Mexico

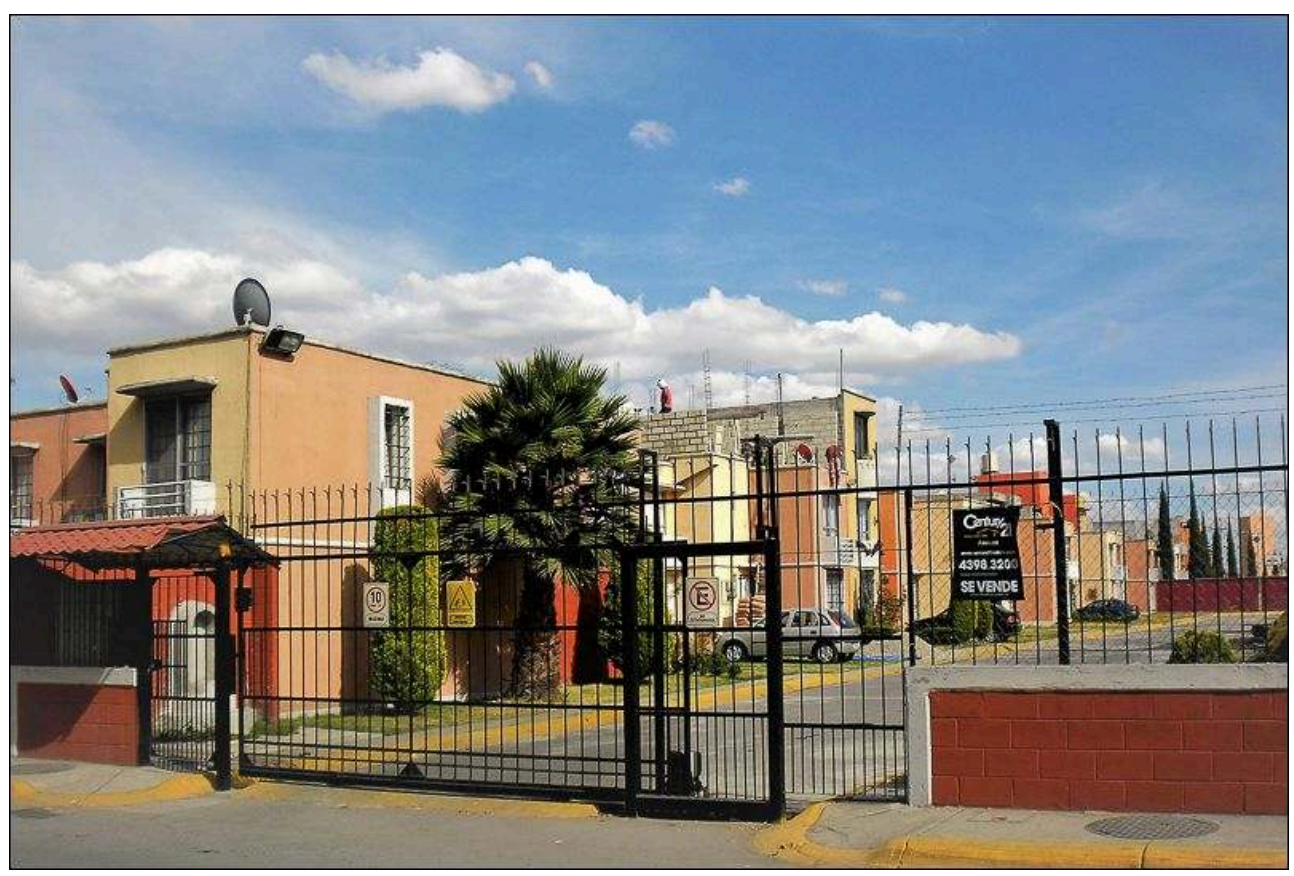

Auteur : J.-F. Valette, novembre 2014

Dans les quartiers enquêtés, le marché immobilier est très visible dans l'espace public : les annonces dans les rues abondent, qu'il s'agisse de reventes de maisons achetées via des crédits aidés, de locations ou sous-locations, ou encore de rachats de crédits. Si les reventes ou les rachats de crédit peuvent faire l'objet de transactions officielles et de gestions par des intermédiaires en règle, visibles par la "qualité » de l'affiche, son caractère informatisé et la mention de logos officiels de crédit - tels que l'INFONAVIT (Institut du Fonds National du Logement pour les Travailleurs) sur l'illustration 8 -, les circulations de biens dans ce marché de logements sociaux peuvent aussi renvoyer à des transactions plus officieuses, moins structurées par des professionnels du marché, telles qu'elles apparaissent sur les affiches placardées dans l'ensemble Santa Elena (illustration 9). 
Illustration 8 - Affiches officielles de renégociation de crédit INFONAVIT, Hacienda Cuautitlán, Cuautitlán, État de Mexico

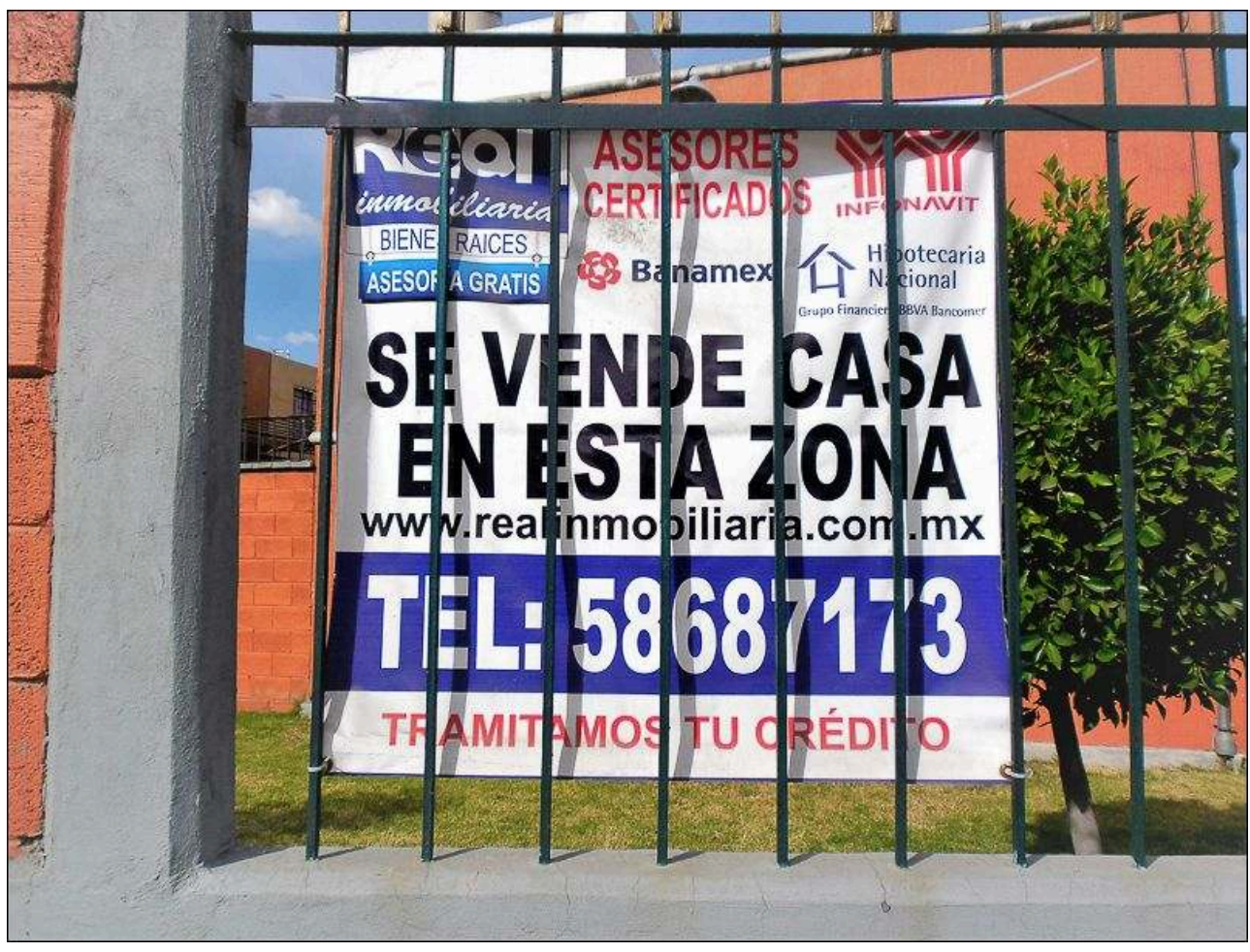

Auteur : J.-F. Valette, novembre 2014

Illustration 9 - Affiches informelles placardées dans les rues du conjunto Santa Elena, Cuautitlán, Etat de Mexico

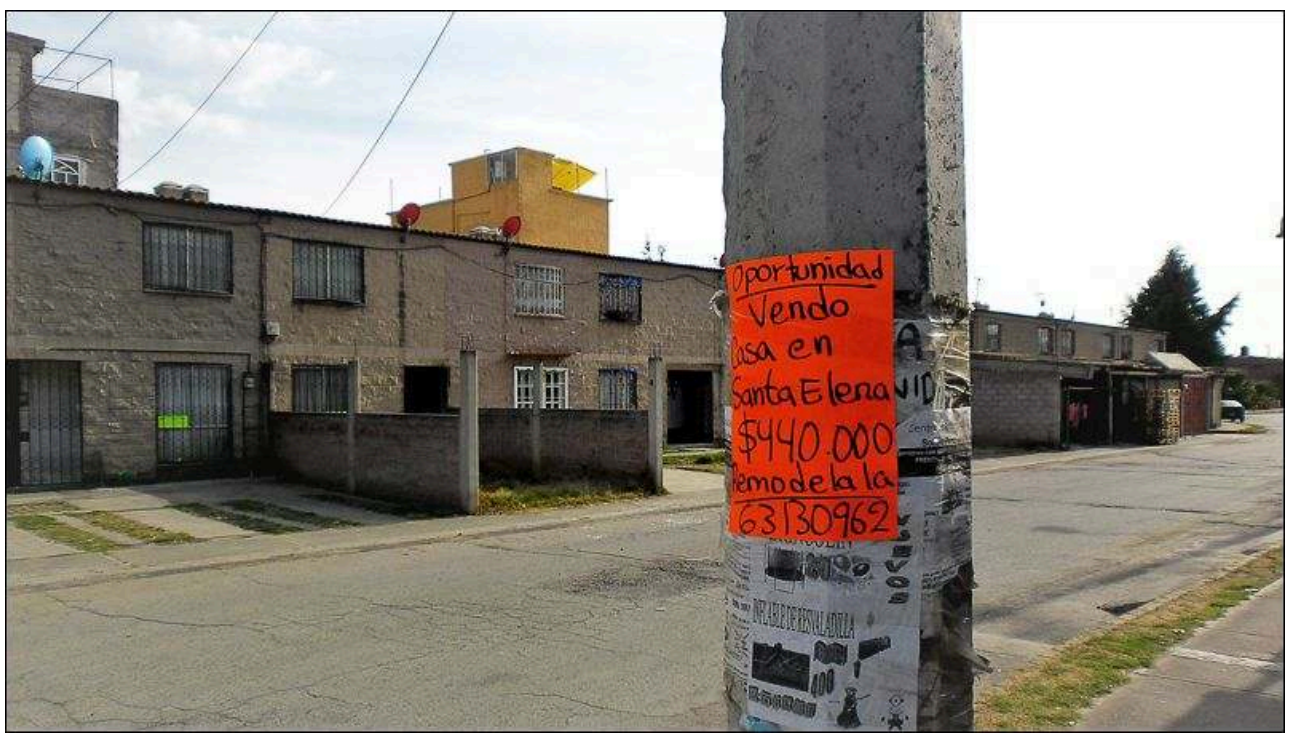

Auteur: J.-F. Valette, novembre 2014

17 La construction de logements a été massive depuis les années 2000, mais le parc n'est pas pour autant «figé », ni dans sa morphologie, ni dans sa composition sociale. Le vieillissement des constructions et la « dé-standardisation » du modèle s'accompagnent de dynamiques de transformations pouvant participer à l'augmentation de la valeur des 
biens : les propriétaires peuvent ajouter des pièces, agrandir la construction (pour un local commercial par exemple), même si ces pratiques sont théoriquement prohibées par les règlements de copropriété.

Illustration 10 - Modifications de la morphologie et de la fonction du bâti dans le conjunto Santa Elena, Cuautitlán, État de Mexico

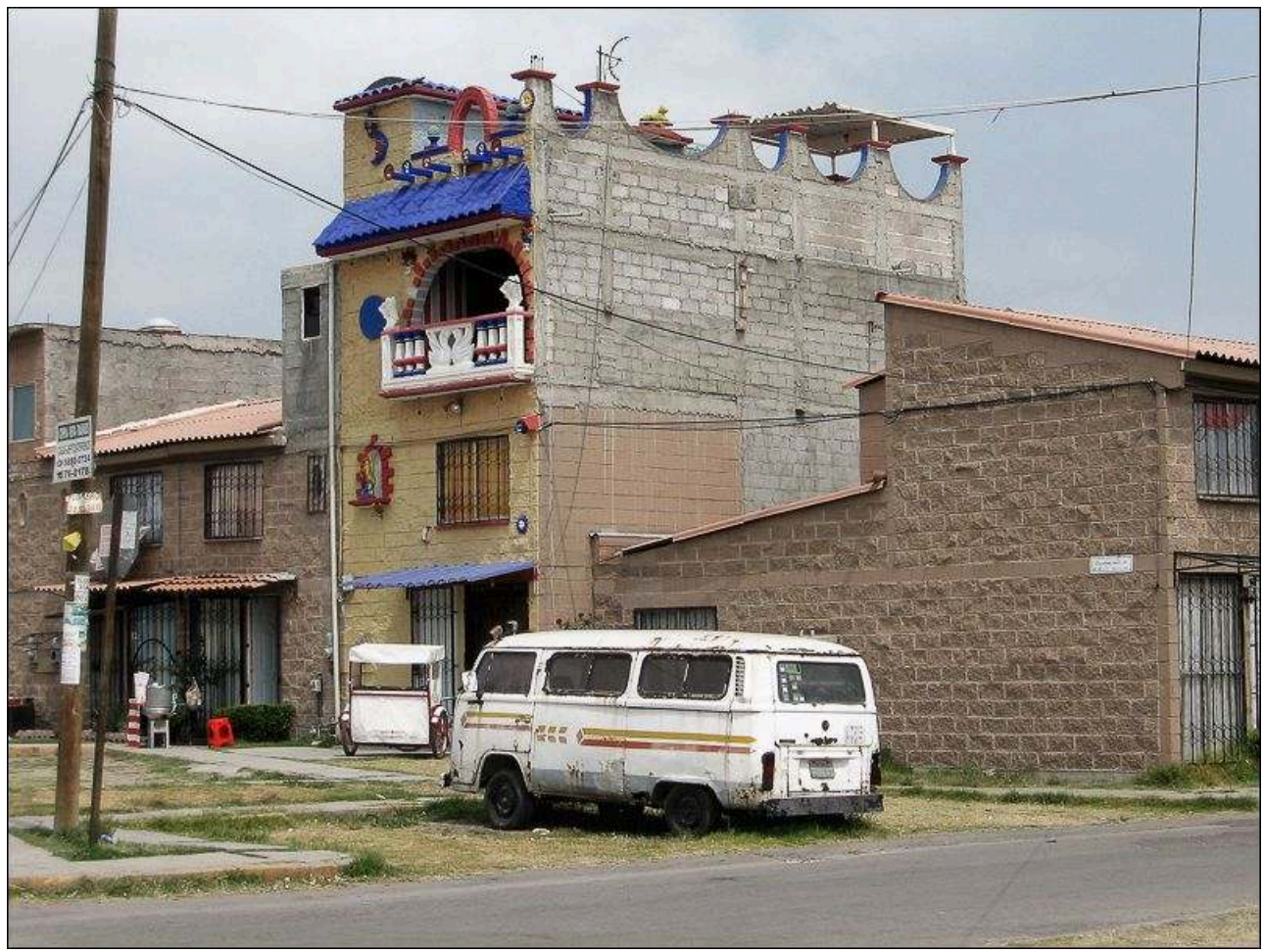

Auteur: J.-F. Valette, novembre 2014

18 Au sein des deux grands parcs de logements populaires, on observe ainsi une grande différence dans la visibilité des marchés fonciers et immobiliers. Les ventes immobilières sont peu visibles dans les colonies populaires les plus récentes, tandis que les locations et surtout l'exploitation de la ressource foncière disponible fonctionnent à plein. Dans les ensembles résidentiels, un bien « d'intérêt social » peut constituer une opportunité d'investissement (par accès au crédit) et faire l'objet de spéculation et/ou de circulation résidentielle multigénérationnelle.

\section{Marchés plus formalisés : la pluralité des canaux d'information}

19 Dans les zones résidentielles des catégories moyennes et aisées, les marchés immobiliers sont caractérisés par une diversité des canaux d'information: en particulier, ce sont ces zones qui sont les mieux représentées sur les portails Internet (Ribardière, Valette, 2017). Toutefois, cette présence en ligne n'obère pas le rôle important joué par les affiches dans l'espace public. Les marchés plus formalisés à destination des catégories moyennes et supérieures renvoient en effet à une publicité importante par ce média, qu'il s'agisse de biens neufs dans les zones denses centrales et 
péricentrales (illustration 11) ou de reventes de maisons-villas dans les zones plus périphériques et plus homogènes de l'ouest de la métropole (illustration 12).

Dans le premier cas, l'exemple dans le quartier moyen-aisé de la Narvarte (délégation Benito Juárez) témoigne de la vitalité du marché, notamment de l'immobilier neuf, dans cette partie péricentrale de la ville qui connait depuis une dizaine d'années un processus de densification et de valorisation important, permis par des politiques urbaines incitatives. La tension vive sur le foncier attise une augmentation des prix, à l'origine d'un filtrage de la population et de l'éviction progressive des catégories modestes (Valdivia, 2014). La surenchère d'annonces de préventes atteste non seulement de l'existence de nombreux programmes immobiliers neufs, mais également de la concurrence que se livrent les promoteurs dans ces espaces stratégiques.

Illustration 11 - Préventes d'appartements et de maisons dans la colonie Narvarte, Benito Juárez, CDMX

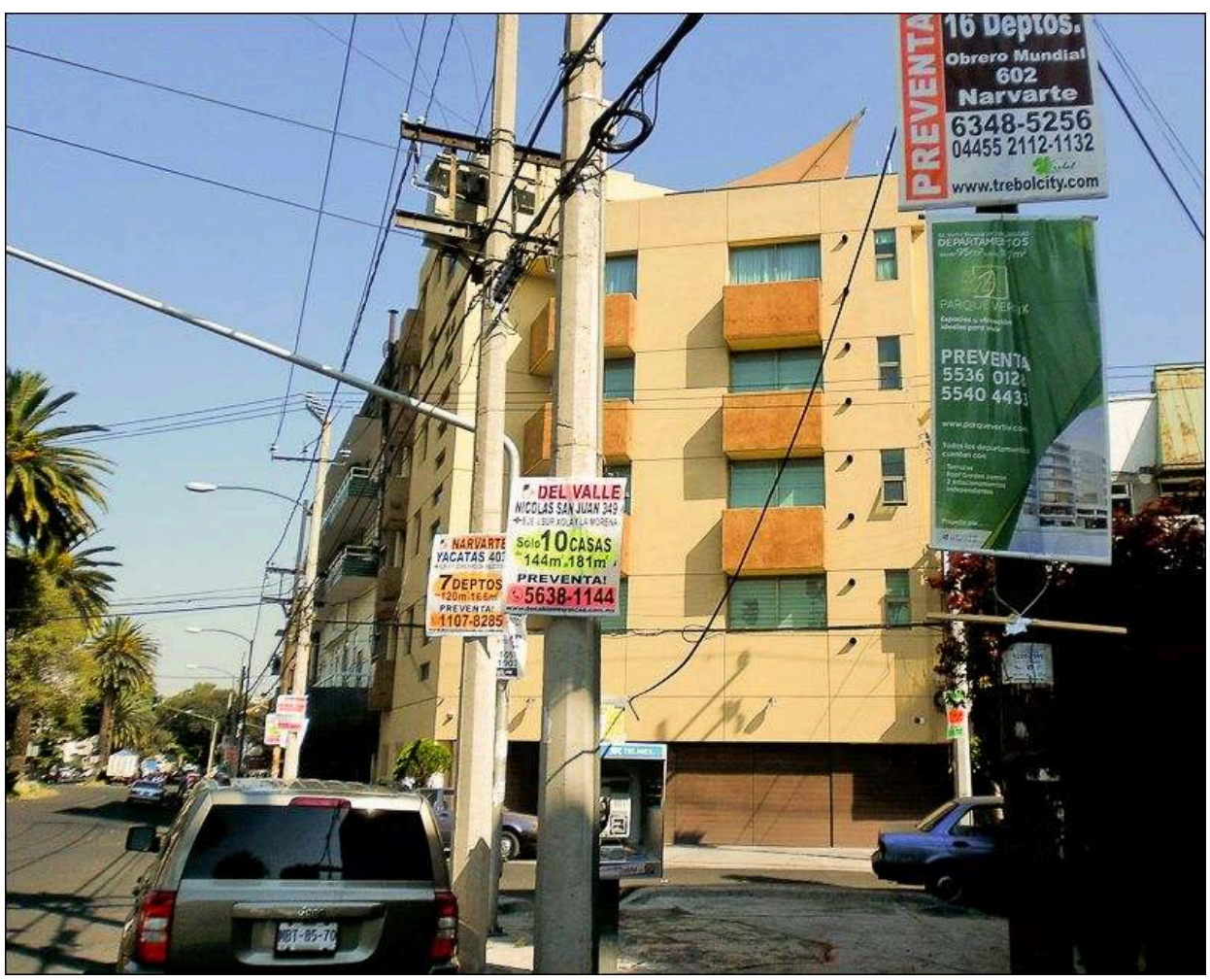

Auteur : J.-F. Valette, novembre 2014

Le second exemple se situe dans le quartier huppé des Lomas de Chapultepec. Même si le marché semble ici plus discret, les annonces affichées sur le mobilier urbain ou directement sur le bien sont pourtant bien présentes. La gamme des prix élevés des ventes n'empêche pas la diffusion de l'information par ces canaux a priori moins formalisés : au contraire, ces cas sont caractérisés par la multitude des médias. À côté des annonces immobilières en ligne, qui maximisent l'intermédiation entre vendeurs et acheteurs ou entre propriétaires et locataires (Larceneux et al., 2014), la diffusion de l'information à une échelle plus locale semble garder tout son intérêt. Au total, la multiplicité des canaux d'information apparaît d'autant plus forte que la valeur du bien est élevée. 
Illustration 12 - Vente d'une maison de luxe dans le quartier des Lomas de Chapultepec, Miguel Hidalgo, CDMX

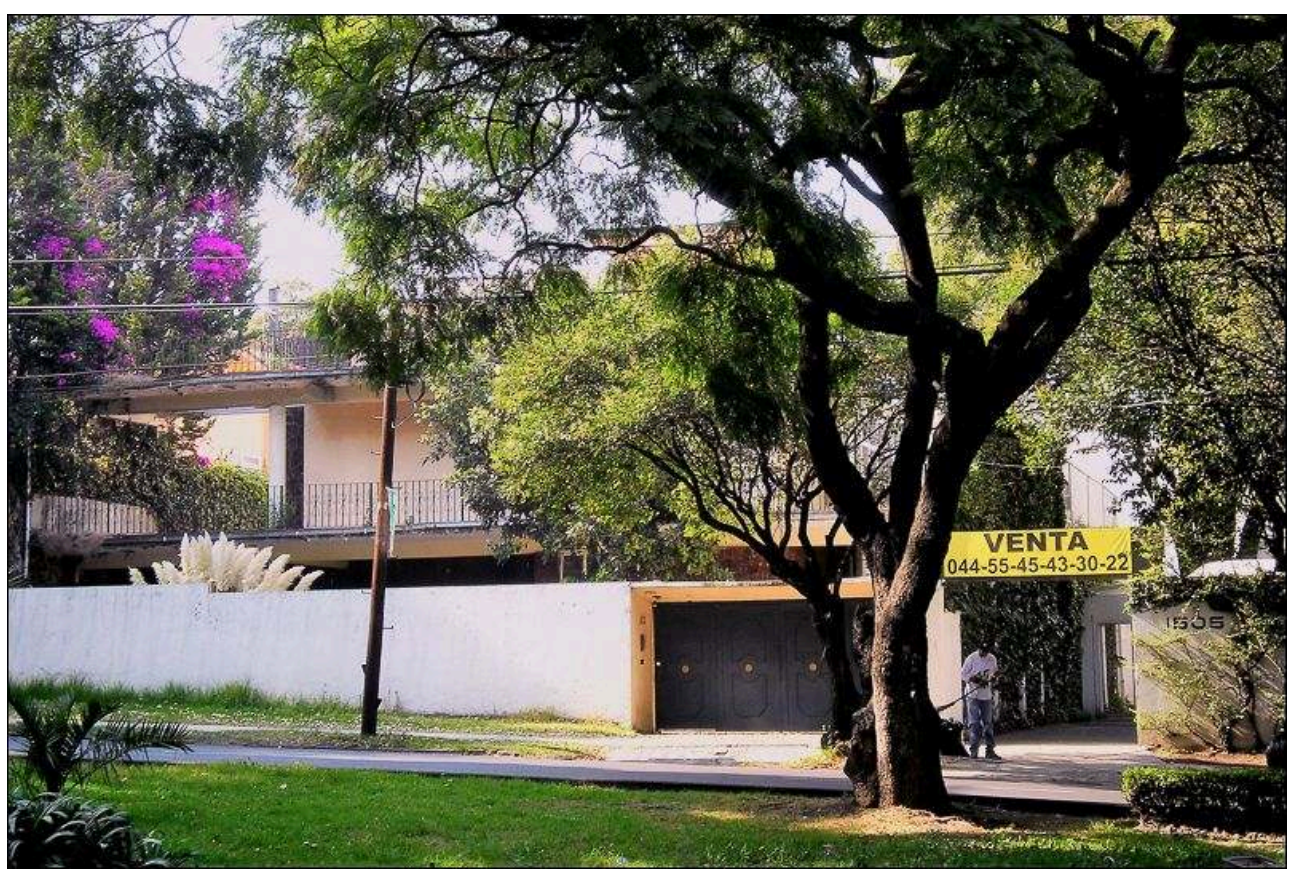

Auteur : J.-F. Valette, novembre 2014

\section{Conclusion}

Les affiches apposées dans l'espace public correspondent à un média incontournable dans la visibilité du marché du logement, comme du marché foncier. Malgré l'existence d'autres supports possibles et le rôle croissant des intermédiaires, en particulier sur Internet, l'affichage dans l'espace public reste d'actualité, quel que soit le contexte résidentiel.

23 L'intérêt porté à ces affiches permet de saisir la prégnance du marché foncier sur les fronts d'urbanisation et les tensions qui y sont associées; la régularisation en marche des colonies populaires consolidées, investies par les agences internationales; la spécificité du peuplement des lotissements géants de logements sociaux, caractérisés par une durée d'installation plus faible qu'en colonie populaire et par des stratégies d'investissement complexes; ainsi que la pression sur les espaces péricentraux, convoités par les promoteurs.

24 Si les affiches sont présentes dans tous les contextes résidentiels, elles s'articulent différemment avec les autres canaux de diffusion, témoignant en cela de la coexistence de filières d'accès au toit et au sol qui restent fort hétérogènes. 


\section{BIBLIOGRAPHY}

Boulay G., Guérois M., Le Goix R., 2011. Acquéreurs et vendeurs dans l'inflation immobilière à Paris et Marseille, 1996-2006. In Pumain D., Mattei M.-F., Données Urbaines, Anthropos /

Economica, Coll. « Villes », p. 167-179.

Driant J.-C., 2006. Quelques enjeux urbains des politiques de l'habitat face aux marchés du logement. Revue d'économie financière, ${ }^{\circ}{ }^{\circ} 86$, p. 199-212.

Duhau E., 2004. Politiques du logement et intégration au Mexique : de la promotion publique à la promotion immobilière privée. Cahier des Amériques Latines, ${ }^{\circ}$ 44, p. 119-136.

Halbwachs M., 1908. La politique foncière des municipalités. Brochure de la librairie du Parti socialiste, $\mathrm{n}^{\circ}$ 3, coll. « Les cahiers du socialiste », Paris, $31 \mathrm{p}$.

Hiernaux-Nicolas D., 1991. La Autoconstrucción de la Vivienda en el Área Metropolitana de la Ciudad de México. Revista Interpretaciones, $n^{\circ}$ 1, México D.F. p. 58-73

Hoyt H., 1939. The Structure and Growth of Residential Neighborhoods in American Cities. Washington, Federal Housing Administration

INEGI, 2016. Encuesta Intercensal 2015. Instituto Nacional de Estadísticas y Geografía, [en ligne] : http://www.beta.inegi.org.mx/proyectos/enchogares/especiales/intercensal/, consulté le 6 mai 2017.

Kunz Balaños I., González Sánchez J., Valverde C. (dir.), 2001. El Mercado inmobiliario habitacional de la Ciudad de México. UNAM, 143 p.

Larceneux F., 2014. La disparition de l'agent immobilier : utopies et réalités de l'intermédiation. L'état des entreprises 2014, Paris, La Découverte, p. 17-30.

Mouillart M., 1998. L'analyse économique du bien logement. In Séagud M., Bonvalet C., Brun J., dir.. Logement et habitat, l'état des savoirs. La Découverte, « Textes à l'appui », Paris, p. 167-176.

Renard V., 2003. Les enjeux urbains des marchés fonciers et immobiliers. In Prager J.-C., Ville et économie, La documentation française, Paris, p. 95-108.

Ribardière A., Valette J.-F., 2017. Géographie des prix immobiliers à Mexico : variabilité et hétérogénéité des valeurs enregistrées dans les annonces en ligne. Cybergeo : European Journal of Geography [en ligne], Espace, Société, Territoire, document 814, URL : http:// cybergeo.revues.org/28040 ; DOI : 10.4000/cybergeo.28040

SEDESOL, CONAPO, INEGI, 2012. Delimitación de las zonas metropolitanas de México 2010, México.

Valdivia R., 2014. Modelo de precios hedónicos para la vivienda nueva en la ZMVM. Estudio de caso en la Zona metropolitana del Valle de México, Publicia, 257 p.

\section{NOTES}

1. Dans le sens où l'affiche ne suppose pas une grande distance entre le lieu où elle est placardée - et en cela, lue par un intéressé potentiel - et la localisation du bien en question : l'annonce peut renvoyer au quartier ou la proximité directe, ou encore au lieu même de l'affichage. 
2. En 2017, la ZMVM compte 76 unités administratives : 16 délégations de la Ciudad de México (ex District Fédéral - DF), 59 municipalités de l'État de Mexico et une de l'État d'Hidalgo (SEDESOL et al, 2012).

3. Les missions 2014 et 2015 ont été réalisées dans le cadre du PICS RESUM financé par le CNRS et dans le cadre du groupe de travail « Produire la ville ordinaire » du LabEx DynamiTe.

4. Au Mexique, il existe trois types de propriété : sociale (la forme la plus répandue étant l'ejido ou la comunidad au statut invendable et inaliénable), publique et privée.

5. Comprises entre 300 pesos $(\mathrm{MXN}) / \mathrm{m}^{2}$ (soit l'équivalent de $20 €$ ) pour un lot « rustique » (sans service) en périphérie du village, et $1000 \mathrm{MXN} / \mathrm{m}^{2}(60 €)$. Dans cet article, les conversions retenues renvoient au niveau du 1er juillet $2014,1 \mathrm{MXN}$ pour $0,06 €$.

6. Les prix des terrains s'élèvent en moyenne ici en 2014 à $210 € / \mathrm{m}^{2}$; en 2010 , il fallait compter aux alentours de $120 € / \mathrm{m}^{2}$. Si ces valeurs restent moindres par rapport aux zones plus péricentrales, elles sont néanmoins très élevées par rapport à d'autres périphéries de la ville.

7. Il faut compter en 2014 dans le quartier Jiménez Cantú entre 25 et $50 € /$ mois, selon la taille de la chambre, les services à disposition (notamment la connexion à l'eau), et la proximité des axes de circulation des transports en commun.

8. Ces stratégies ne sont pas exclusives aux colonies populaires d'une part, la production résidentielle des colonies populaires, même si elle en est fortement marquée, ne se réduit pas à pas ce seul mode d'autre part. 